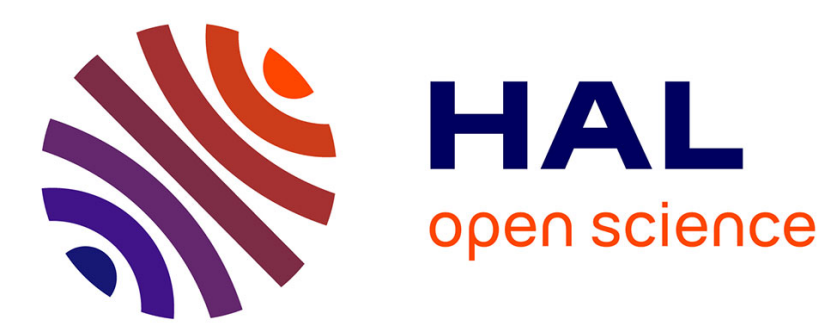

\title{
On Probabilities in Biology and Physics
}

Joseph Berkovitz, Philippe Huneman

\section{To cite this version:}

Joseph Berkovitz, Philippe Huneman. On Probabilities in Biology and Physics. Erkenntnis, 2015, 80 (S3), pp.433-456. 10.1007/s10670-015-9780-8 . hal-01968503

\section{HAL Id: hal-01968503 https://hal.science/hal-01968503}

Submitted on 10 Jan 2019

HAL is a multi-disciplinary open access archive for the deposit and dissemination of scientific research documents, whether they are published or not. The documents may come from teaching and research institutions in France or abroad, or from public or private research centers.
L'archive ouverte pluridisciplinaire HAL, est destinée au dépôt et à la diffusion de documents scientifiques de niveau recherche, publiés ou non, émanant des établissements d'enseignement et de recherche français ou étrangers, des laboratoires publics ou privés. 


\section{On Probabilities in Biology and Physics}

\section{Joseph Berkovitz \& Philippe Huneman}

\section{Erkenntnis}

An International Journal of Scientific

Philosophy

\section{ISSN 0165-0106}

Volume 80

Supplement 3

Erkenn (2015) 80:433-456

\section{E R K E N N T N I S}

DOI 10.1007/s10670-015-9780-8

AN INTERNATIONAL JOURNAL OF

SCIENTIFIC PHILOSOPHY

Editor-in-chief: Hannes Leitgeb

ON PROBABILITIES IN BIOLOGY AND PHYSICS

Guest Editors:

Joseph Berkovitz

and Philosophy of Science and Technology, University of Toronto

Philippe Huneman

Institut d'Histoire et de Philosophie des Sciences et des Techniques, CNRS/Université Paris I Sorbonne

\section{Springer}


Your article is protected by copyright and all rights are held exclusively by Springer Science +Business Media Dordrecht. This e-offprint is for personal use only and shall not be selfarchived in electronic repositories. If you wish to self-archive your article, please use the accepted manuscript version for posting on your own website. You may further deposit the accepted manuscript version in any repository, provided it is only made publicly available 12 months after official publication or later and provided acknowledgement is given to the original source of publication and a link is inserted to the published article on Springer's website. The link must be accompanied by the following text: "The final publication is available at link.springer.com". 


\title{
On Probabilities in Biology and Physics
}

\author{
Joseph Berkovitz ${ }^{1}$ Philippe Huneman ${ }^{2}$
}

Received: 22 September 2015/Accepted: 24 September 2015/Published online: 25 November 2015

(C) Springer Science+Business Media Dordrecht 2015

\begin{abstract}
This volume focuses on various questions concerning the interpretation of probability and probabilistic reasoning in biology and physics. It is inspired by the idea that philosophers of biology and philosophers of physics who work on the foundations of their disciplines encounter similar questions and problems concerning the role and application of probability, and that interaction between the two communities will be both interesting and fruitful. In this introduction we present the background to the main questions that the volume focuses on and summarize the highlights of the individual contributions.
\end{abstract}

\section{The Background and Motivation}

Probability is ubiquitous in modern science. While there is a broad consensus about the axiomatic theory of probability, there is an ongoing discussion and controversy over the interpretations of probability in scientific theories and models. Yet, the interpretation of probability and probabilistic reasoning are central to the understanding of scientific theories, models and practices. This volume focuses on various questions concerning the interpretation of probability and probabilistic reasoning in biology and physics. It is the outcome of a 3-day workshop on Probability in Biology and Physics at the IHPST, Paris, which took place on February 12-14, 2009. The idea of the workshop was that philosophers of biology and philosophers of physics who work on the foundations of their disciplines

Joseph Berkovitz

joseph.berkovitz@utoronto.ca

1 IHPST, University of Toronto, Victoria College, 91 Charles St. West, Toronto, ON M5S 1K7, Canada

2 Institut d'Histoire et de Philosophie des Sciences et des Techniques, CNRS/Université Paris I Sorbonne, Paris, France 
encounter similar questions and problems concerning the role and application of probability, and that interaction between the two communities will be both interesting and conducive. The discussions were lively and fruitful and a joint effort has led to the birth of this volume. Since the Paris workshop, there were two other successful meetings that brought together philosophers of biology and philosophers of physics to discuss questions concerning causality, explanation and time in biology and physics: a 3-day conference on Causality and Explanation in Physics, Biology and Economics in Barcelona on February 18-20, 2010, ${ }^{1}$ and a 3-day workshop, The Time of Nature, The Nature of Time in Bordeaux on September 26-28, 2012. ${ }^{2}$ It is our hope that this volume will continue this trend and communicate to a larger audience of philosophers some of the outcomes of these interactions.

Many philosophers of science are familiar with the philosophical foundations of probability. Yet, in the interest of appealing to a broader audience, we start with a brief, uncritical and non-exhaustive introduction to the various interpretations of probability that play a role in science (Sect. 2). ${ }^{3}$ We then give some examples of questions concerning the interpretation of probability and its application in biology and physics (Sect. 3). Some of these examples draw attention to similar questions and problems that arise in the application of probability in both fields. We conclude with a background and overview of the papers in this volume (Sect. 4).

\section{A Brief Sketch of Interpretations of Probability}

Interpretations of probability are commonly divided into two main groups: objective and subjective. Subjective probabilities are supposed to reflect rational degrees of belief about things one is uncertain about. De Finetti held that for degrees of belief to be rational they have to be coherent, and for degrees of belief to be coherent they have to be probabilities, i.e. they have to satisfy the probability calculus (de Finetti 1972, 1974a, b). Ramsey held a similar view, namely that for degrees of belief to be rational they have to be consistent, and that for degrees of belief to be consistent they have to be probabilities (Ramsey 1926/1990). ${ }^{4}$ Subjective probability interpretations vary according to the extent to which probability is subjective. The most radical subjective interpretation, de Finetti's (1972, 1974a, b) influential theory of probability, denies the existence of objective probability. It is common to portray probability in de Finetti's subjective theory of probability as unconstrained, too permissive and possibly whimsical (see, for example, Hájek 2012, Sect. 3.5.4). But such a portrayal fails to distinguish between the ontological status of probability and the way it should be evaluated (Berkovitz 2012, Sect. 16.3.4, 2014). De Finetti was an instrumentalist about probability. He held that ontologically probabilities are

\footnotetext{
${ }_{1}$ http://conectahistoria.blogspot.ca/2010/02/barcelona-conference-on-causality-and.html.

2 http://www.msha.fr/msha/actu/colloque/pages/presentation_time_of_nature.pdf.

3 For more detailed reviews of interpretations of probability, see Gillies (2000a), Galavotti (2005) and Hájek (2012).

${ }^{4}$ In the literature, it is often assumed that by coherence de Finetti means consistency (Howson 2008; Dickey et al. 2009; Vineberg 2011), but Berkovitz (2014) argues that this assumption is unjustified.
} 
subjective, reflecting one's ignorance, but that their assignment should not be arbitrary. Indeed, de Finetti held that assigning and evaluating probabilities is a form of inductive reasoning, and as such should not be the result of a capricious psychological reaction, "but as a mental process susceptible of an analysis, interpretation and justification" (de Finetti 1972, p. 147). ${ }^{5}$

Other subjective theories of probability acknowledge the existence of objective probabilities and require that subjective probabilities of events be constrained by the relevant objective probabilities, whenever such objective probabilities are available and provide the only information about the prospects of these events. For example, it is common to assume the following principle: the subjective probability of an event $E$ given that the objective probability of $E$ is $p$, and there is no other information about the prospects of $E$, should be $p$. Hacking's (1965, chap. 9) frequency principle, Lewis' (1986, chap. 19) principal principle and Mellor's (1995, chap. 4) evidence condition are variations of such a principle. For a discussion of the principal principle, see Frigg and Hoefer (2013).

Some subjective interpretations of probability also require that the assignments of probabilities should be subjected to other principles. Notable examples are principles of indifference: "if no known reason for predicating of ... one rather than another of several alternatives, then relatively to such knowledge the assertions of each of these alternatives have an equal probability" (Keynes 1921/1963, p. 42). The principle of maximum entropy, introduced by Jaynes (1957), is a generalization of Keynes' indifference principle to non-uniform probability distributions. Interpretations of probability that impose the principle of maximum entropy and similar principles to determine prior probabilities are sometimes called 'objective Bayesian'. The objective nature of probabilities in these interpretations is questionable, however, as the motivation for principles of indifference is characteristically based on ignorance rather than objective facts.

Objective probabilities are supposed to reflect objective facts about the world. Among the popular contemporary objective interpretations of probability are frequency theories, propensity theories, theories of Humean objective chance (THOC) and the so-called 'range conception' theories.

Frequency theories explicate probabilities of events in terms of their actual or hypothetical, finite or infinite relative frequencies in given reference classes. ${ }^{6}$ For example, the probability of a certain coin landing on heads in the reference class of

\footnotetext{
5 The conflation of the ontological status of theoretical terms with the way they are to be evaluated and their values as instruments is not particular to the interpretation of de Finetti's theory (Berkovitz 2014). In discussions of instrumentalism it is common to associate the instrumental value of theoretical postulates with their ontological status. Thus, for example, it is argued that under instrumentalism, theories are capable (at best) of accommodating known observable phenomena, and incapable of making novel predictions. Psillos (1999, p. 29) interprets Duhem as arguing along these lines. "Duhem's point is that the fact that some theories generate novel predictions cannot be accounted for on a purely instrumentalist understanding of scientific theories. For how can one expect that an arbitrary (artificial) classification of a set of known experimental laws - i.e. a classification based only on considerations of convenience - will possibly be able to reveal unforeseen phenomena in the world?" The presupposition is that the ontological status of theoretical terms determines their capacity to generate novel predictions. But this presupposition begs the question against instrumentalism in general and de Finetti's instrumentalism in particular (Berkovitz 2014).

6 For the sake of brevity, in what follows by 'frequency' we will mean relative frequency.
} 
a given sequence of tosses of this coin is the relative frequency of heads in that reference class. Intuitively, not any frequency will be considered worthy of the title probability. If the pattern of heads in the reference class is predictable, e.g. if the coin lands on heads every other toss, the frequency of heads will be 0.5 . But such a sequence would not be suitable for characterizing probability, as probability is associated with randomness. Indeed, it is a fundamental tenet of objective probability that the products of probabilistic processes are random. ${ }^{7}$ Thus, frequency theorists explicate probability in terms of frequencies in random sequences. The scope of frequency theories is quite broad: they are applicable to the interpretations of probabilities in both deterministic and indeterministic scientific theories. Notable frequency theorists are Ellis (1849), Venn (1866), von Mises (1928/ 1962) and Reichenbach (1949), though von Mises' theory is the best known and most developed.

In propensity theories, probabilities are explicated in terms of dispositions or tendencies that are properties of, or are related to, objects, experimental arrangements or physical set-ups. Propensity theories may be divided into two main groups: long-run and single-case. In long-run propensity theories, propensities are explicated in terms of long-run frequencies (Peirce 1910/2011; Popper 1957, 1959; Hacking 1965; Gillies $2000 \mathrm{a}, \mathrm{b})$. The characterization of long-run propensity varies from one theory to another. Peirce, the anticipator of the propensity interpretation, characterized probability as a "would be" of an object to yield a certain frequency of events in an infinite series of independent trials of the same kind. For example, certain coins have a "would be", or in current terminology a propensity to yield a long-run frequency 0.5 to land on heads. Popper (1957, 1959), who proposed the propensity interpretation, presented it in an ambiguous way. On the one hand, propensity is characterized in terms of long-run frequency: it is a propensity of an experimental arrangement (rather than just an object in it) to yield a frequency of results when the experiment is often repeated, though it is not clear whether the long-run is finite or infinite. But, on the other hand, propensity is characterized in terms of the single-case: it is a propensity of an experimental arrangement to realize a singular result. Hacking (1965) takes propensity to be a disposition of a kind of trial on a chance set-up to yield a long-run frequency, where 'long-run frequency', 'chance set-up' and 'trial' are primitive terms that are characterized by various postulates. Gillies (2000a) regards repeatable conditions as having a propensity to produce frequencies which are approximately equal to the long-run propensity, where the approximation is characterized by a falsifying rule for statements about long-run propensities. Both Hacking's and Gillies' theories are finite long-run propensity theories: the frequencies that explicate propensity are finite.

Single-case propensity theories may be divided into two kinds: tendency theories in which propensities are probabilities (Popper 1957, 1959, 1990; Giere 1973a, b, 1975, 1979; Fetzer 1974, 1981, 1982; Miller 1991, 1994), and dispositional theories in which propensities are not probabilities but rather dispositions to display probability distributions (Mellor 1969, 1971, 2005; Suarez 2013). In tendency theories of single-case propensity, the propensity of an event is thought of as a

\footnotetext{
7 For a discussion of this tenet in the context of long-run propensity theories, see Berkovitz (2015, Sect. 3.5).
} 
single-case tendency or 'indeterministic disposition' of properties of, or trials on the relevant physical set-up to yield the event; where the tendency and disposition involved embody causal efficacy. The various tendency theories vary in their characterization of the relevant set-up. Giere (1973a) characterizes propensities as tendencies of 'whole set-ups' to produce various outcomes on particular trials. Popper (1990) takes propensity to be a tendency of the 'whole physical situation' to realize a particular event. Similarly, Miller (1994) suggests that, strictly, any propensity must be referred to the 'complete situation of the universe at the time'. For Fetzer (1982), a single-case propensity of an event is a tendency of a complete set of nomically and/or causally relevant conditions to yield the event. ${ }^{8}$ In dispositional theories of single-case propensity, a propensity is a 'sure-fire' disposition that is attributed to a part of the relevant physical set-up and its displays is a single-case objective probability distribution, the 'chance distribution', of the possible outcomes of trials on the entire set-up (Mellor 1969, 1971; Suarez 2013). Mellor's theory is based on epistemic foundations. Mellor (1971) holds that to ascribe a chance to an outcome of a trial is no more than to restate that there is an objective constraint on the partial belief reasonably held on the trial's occurrence, and the constraint is a propensity that is attributed to part of the entire relevant physical set-up. Suarez's theory is inspired by Peirce's pragmatist philosophy and it is not based on epistemic foundations. ${ }^{9}$

Like frequency theories, long-run propensity theories apply to both indeterministic and deterministic theories. By contrast, single-case propensity theories apply only to indeterministic theories.

In THOC, probabilities are objective. But, Lewis, the founding father of THOC, held that subjective probability is the best guide for Humean objective chances (HOC). He introduced HOC by the principal principle (PP), ${ }^{10}$ and he argued that PP captures all that we know about HOC (Lewis 1986, chap. 19). Like single-case propensities, Lewis conceived HOC as objective single-case probabilities that apply only to the interpretation of probabilities in indeterministic theories. But, unlike single-case propensities, HOC supervene upon the mosaic of matters of particular facts about objects and spacetime regions of the universe. The main idea is that laws of HOC and rules for their assignments are part of a 'best system' of rules and laws that strikes the best combination of, or balance between, simplicity, strength and fit with actual matters of fact; and given such a balance, laws and rules concerning HOC supervene upon matters of particular fact.

Some versions of THOC differ from Lewis' in important respects. In particular, Frigg and Hoefer (2013) propose a version of THOC that can be utilized in both

\footnotetext{
${ }^{8}$ However, his theory cannot be considered as an interpretation of the probability calculus since it violates it.

9 For a detailed discussion of propensity theories, see Berkovitz (2015).

${ }^{10}$ Lewis (1986, p. 87) formulates the principal principle as follows: "Let $C$ be any reasonable initial credence function. Let $t$ be any time. Let $x$ be any real number in the unit interval. Let $X$ be the proposition that the chance, at time $t$, of $A$ 's holding equals $x$. Let $E$ be any proposition compatible with $X$ that is admissible at time $t$. Then $C(A / X E)=x$." $C$ is a non-negative, normalized, finitely additive measure defined on all propositions (sets of worlds), and $E$ is admissible at time $t$ if it contains only information whose impact on the credence of $A$ comes entirely by way of credence about the chance of $A$.
} 
indeterministic and deterministic theories and then apply it to the interpretation of the probabilities in Boltzmannian statistical mechanics.

Another kind of objective interpretation of probability is situated in the tradition of von Kries' (1886) "Spielraumtheorie" and Poincaré's (1912) method of arbitrary functions. Rosenthal (2012) calls this interpretation the 'range conception' of probability, echoing von Kries' terminology. Versions of the range conception interpretation have been proposed by Strevens (2003, 2011), Rosenthal (2010, 2012) and Abrams (2012). The interpretation is mainly intended to apply to deterministic macroscopic processes. ${ }^{11}$ It explicates the probability of an event-an 'outcome'in terms of the ratio of the input states that lead to it. Following Strevens' (2011) terminology, the basic idea is the following. Imagine a space of initial states of a given deterministic process. Each point in this space represents a maximally specific state the process could start out, and accordingly it leads to a certain outcome. Suppose that the dynamics of the process is such that in each 'small' but 'not too small region' of this input space, the ratio of states that leads to a given outcome (henceforth, the strike ratio with respect to that outcome) is the same (henceforth, microconstant dynamics). Suppose further that the input distribution possesses a certain smoothness property (henceforth, macroperiodicity), so that it is roughly flat over any small interval. In a microconstant dynamics with an input distribution that is macroperiodic, the probability of an outcome is equal to its strike ratio.

The nature of range-conception probabilities depends on the interpretation of the input probability distributions. Strevens (2011) suggests that the input probability distribution is the one that summarizes best the actual frequencies of initial states. If there are no such actual frequencies, the input probability distribution is the one that summarizes best the frequencies of the initial states in almost all the possible worlds in which the relevant actual set-up occurs. Strevens (2011, Sect. 4) maintains that the input distributions do not need a probability interpretation, and he rejects the idea that they should be interpreted along the frequency interpretation because they are not probability distributions but rather non-probabilistic facts about actual or hypothetical frequencies, which depend on the relevant actual set-up. Similarly, Abrams (2012) thinks of the input distribution as a probability measure that reflects facts about actual frequencies of initial states which depend on the relevant actual set-up. Rosenthal takes range-conception probabilities to be non-single-case objective probabilities that are features of experimental set-ups which explain the characteristic frequencies of the various outcomes. Rosenthal (2012, Sect. 4) does not interpret the input probabilities. He argues that we need not assume that there is a probability distribution over the space of input states according to which nature selects initial states because under certain conditions (which correspond to the ones described above) the outcome probabilities do not depend on the probability distribution over the input-state space. It is the structure of the input space that determines the outcome probabilities.

In the literature, there are also epistemic and logical theories of probabilities. Arguably, epistemic theories of probabilities may be included within the range of

\footnotetext{
${ }^{11}$ Unlike Strevens and Rosenthal, Abrams intends his interpretation to apply to both deterministic and indeterministic processes.
} 
subjective theories of probabilities, though supporters of epistemic probabilities tend to emphasize their non-subjective characteristics, e.g. principles and considerations that relate them to objective facts and empirical data. Logical probabilities are not supposed to be subjective. Logical theorists of probability maintain that probability can be regarded as a generalization of the notion of logical implication or validity. Thus, for example, the probability of $A$ given $B$ is explicated as the degree to which $A$ is valid relative to $B$ (Bolzano 1973), the degree to which $B$ implies $A$ (Keynes 1921/1963) or the degree to which $B$ confirms $A$ (Carnap 1950). Supporters of the logical theory of probability argue that it could be the foundation for confirmation theory and more generally 'inductive logic'. Carnap (1950) presents the most systematic development of this view.

Finally, an important interpretative question concerning probability, which pertains to all interpretations, is whether the fundamental object of probability theory is unconditional or conditional probability. Following Kolmogorov (1933/ 1950), it is common in the literature to take unconditional probability as the fundamental object of probability theory, and to define conditional probability in terms of a ratio of unconditional probabilities. But various notable theorists, like Peirce (1910/2011), Johnson (1932), Keynes (1921), de Finetti (1974a, b), Carnap (1952), Popper (1959) and Jeffreys (1961), held that the fundamental concept of probability is conditional probability. Hájek (2003) and Berkovitz (2015, Sect. 5.2) argue that in all the major theories of probability, conditional probability should be the fundamental object and unconditional probabilities do not make sense. For example, it is common to characterize subjective probability as dependent on one's background knowledge. Yet, if subjective probabilities are represented by standard conditional probabilities, as it is common in the literature, we have an infinite regress. The probability of an event $A$ in the background knowledge $B$ is represented by the standard conditional probability of $A$ given $B .^{12}$ And for this probability to be definite, the background knowledge $B$ has to be defined relative to another background knowledge $B^{*}$, i.e. it has to be represented by the standard conditional probability of $B$ given $B^{*}$, and so forth. For another example, propensities are defined relative to conditions that determine them. In the literature it is common to represent the propensity of an event $E$ as the standard conditional probability of that event given the conditions $C$ that determine this propensity. This representation presupposes that $C$ has a definite propensity. But $C$ has definite propensity only relative to some other conditions $C^{*}$, and so we encounter a similar regress. Various authors argue that the question of the nature of the conditional probability that represents propensity is central to the resolution of Humphreys' $(1985,2004)$ paradox, one of the main challenges for propensity theories of probability. ${ }^{13}$

\footnotetext{
12 The standard conditional probability of $A$ given $B$ is defined as the ratio of the unconditional probability of $A \& B$ to the unconditional probability of $B: P(A \& B) / P(B)$.

13 For a discussion of these arguments, see Berkovitz 2015, Sects. 5.2-5.3 and references therein.
} 


\section{On the Interpretation of Probability and Probabilistic Reasoning in Biology and Physics}

The interpretation of probability plays an important role in our understanding of biology and physics. Here are some examples.

One of the main foundational questions in quantum mechanics (QM) is the interpretation of probabilities. This question is intimately related to the interpretation of the representational content of QM wavefunctions. For example, if we interpret QM wavefunctions as reflecting the properties of physical systems, it is natural to interpret QM probabilities along objective theories of probability. It is noteworthy that Popper proposed the propensity interpretation in order to "eliminate from quantum theory certain disturbing elements of an irrational and subjectivist character" (Popper 1959, p. 31), which he attributed to the Bohr-Heisenberg interpretation of QM, and to resolve some of the theory's foundational problems (Popper 1967). ${ }^{14}$ By contrast, if we interpret QM wavefunctions as reflecting knowledge or ignorance about the properties of physical systems, it is natural to interpret QM probabilities along subjective theories of probabilities, as it is common in the increasingly influential quantum information and quantum computing literature. ${ }^{15}$

Questions concerning the interpretation of probabilities are also central to foundational problems in classical statistical mechanics (CSM), a theory that relates Thermodynamics (TD) to classical mechanics (CM). The behavior of macroscopic systems, like a gas in a box, is to a large degree of approximation described correctly by TD. TD introduces macrostates that pertain to a physical system as a whole without any regard to the microscopic makeup of the system. The most important law of TD is the so-called Second Law, stating that the thermodynamic entropy of an isolated system cannot decrease. But there is an entirely different way of looking at the gas in a box, i.e. as a collection of molecules. In this way of looking at the gas the focus is on the microstates of the gas, which are constituted by the molecules' positions and momenta and are governed by the laws of CM. CSM is supposed to establish a connection between the way TD and the way CM describe macroscopic systems and to account for the thermodynamic behaviour of macroscopic systems in terms of both the dynamical laws governing the microscopic constituents of these systems and some probabilistic assumptions. Now, in CM isolated physical systems could evolve into states with lower entropy, and one of the most puzzling questions in the foundations of CSM is why macroscopic systems never seem to evolve into lower-entropy states. Another way to express this puzzle is the question: how could expressly time-asymmetric behaviour of irreversible thermodynamic processes be reconciled with the underlying time-reversible dynamics of CM? The standard answer in CSM is given in terms of probability, namely that thermodynamic behaviour is highly likely, and the question arises as to the meaning of this probabilistic statement. Since CSM is a deterministic theory, it is natural to think of this likelihood as reflecting ignorance

\footnotetext{
14 For a critical discussion of this resolution, see Bub (1975) and Bub and Pitowsky (1985).

15 For examples of subjective interpretations of QM probabilities, see Caves et al. (2002a, b, 2007), Pitowsky (2003) and Berkovitz (2012).
} 
about the initial states of the microsystems. But, this response is controversial and it raises an immediate question: how can our ignorance explain the objective thermodynamic behaviour of systems? Further, in CSM macrostates are sets of microstates and accordingly the probabilities of macrostates are determined by the probability measure over microstates and there is the challenge of justifying this measure, which according to the orthodox wisdom is the Lebesgue measure. As we shall see below, three of the papers in this volume address the question of the explanation of the irreversible behaviour of macroscopic systems and the related question of the interpretation of the probabilities involved.

Questions concerning the interpretation of probability are also prevalent in the biological sciences, and especially in evolutionary biology. Classical evolutionary theory has been developed in the framework of the "Modern Synthesis", i.e. the synthesis of Darwin's theory of natural selection and Mendel's theory of inheritance. It is framed in probabilistic terms. Its core, population genetics, studies the process of evolution focusing on changes in frequencies of alleles in a population. The development of population genetics in the 1920s-1930s was led by Fisher, Haldane and Wright through the use of statistical methods, including methods that were invented by Wright and Fisher. From the onset, an affinity with CSM was recognized (Morrison 2002): CSM studies 'populations' of particles moving in space and population genetics studies populations of entities (alleles) changing their frequency according to some replication rules (i.e. ensembles of individuals moving in a genotype space). Indeed, in his groundbreaking book The genetical theory of natural selection, Fisher acknowledged this proximity. He thought that, in the same way as CSM accounts for the second law of thermodynamics, population genetics could ground a universal law, the "Fundamental Theorem of Natural selection" (FTNS) (1930, p. 36), which is supposed to have a fundamental status corresponding to the status that the Second Law has in TD (though, unlike the Second Law, it has always been controversial ${ }^{16}$ ). Thus, it may not be surprising that philosophers of biology have referred to CSM and TD in controversies about the probabilistic nature of fitness and selection (see, for example, Matthen and Ariew 2002; Bouchard and Rosenberg 2004) and that recently some biologists appealed to these theories in their attempts to reformulate population genetics and quantitative genetics (Ao 2005; Sella and Hirsh 2005; Barton and Coe 2009).

The idea of natural selection in evolutionary biology is that heritable traits that make their carriers more likely to have more offspring in the next generation will very probably increase in frequency, ${ }^{17}$ and a change in the frequency of the traits might also lead to a change in the nature of the traits themselves. Indeed, the main terms of this theory, such as 'fitness' (a measure of the evolutionary success of different individuals, traits or alleles), 'heritability' (which is defined in terms of variances) and 'random genetic drift' (variations in frequency of alleles or traits that

\footnotetext{
${ }^{16}$ FTNS states that "the rate of increase in fitness of any organism at any time is equal to its genetic variance in fitness at that time" (Fisher 1930, p. 35). That is, a population's rate of change in mean fitness due to natural selection is equal to the additive genetic variance. It is important to emphasize here the fact that the variation rate of mean fitness necessarily increases, so the principle singles out a trend in nature, like the Second Law in TD.

${ }^{17}$ In the classical view, this process happens primarily at the level of alleles.
} 
are not related to their adaptedness ${ }^{18}$ ), are probabilistic. Thus, the interpretation of the various probabilities that figure in evolutionary biology has important implications for the representational content of this theory. For example, if random genetic drift is measured in terms of subjective probabilities, then it represents a degree of ignorance about what is actually adaptive. Thus, if we had a better knowledge of the actual interactions between organisms up to their molecular details, there would be less drift: what we deemed random drift would be ascribed to selective pressures that were previously unknown. Similarly, the nature of selection itself may depend upon the interpretation of the probabilistic terms involved in fitness. If the probability distribution of same-type offspring in the next generation for a given type of trait or allele were subjective, it would entail that fitness, and accordingly natural selection, depend on our ignorance, which would be reduced if we knew all about the physical and chemical processes involved (Rosenberg 1994). On the other hand, if we interpret this probability as objective, fitness and selection would represent objective properties of the processes involved.

Probability also plays an important role in causal reasoning in modern science. The question of the relationships between probability and causality is present explicitly or implicitly in almost all the papers in this volume. Four papers concentrate on the foundations of evolutionary biology and debate whether it is appropriate to understand fitness and selection in causal terms and accordingly interpret the probabilities involved along propensity theories of probability. One paper discusses the question whether records of CSM systems could be considered veridical, and the discussion involves presuppositions about the relationships between causation and probability. Another paper analyzes the circumstances under which it is reasonable to suppose that causally related events are probabilistically independent, with a focus on physical probability distributions that are presumably grounded in, among other things, facts about causal relations. Finally, there is a paper that studies the philosophical foundations of propensity theories of probability, and one of the main foundational questions is whether propensity is to be explicated in causal terms.

\section{The Papers}

\subsection{Fitness, Selection and Probability in Evolutionary Biology}

A quick overview of the debates that have occupied philosophers of biology since the academic emergence of the discipline in the 1970s may be divided into three main related topics of controversy concerning probabilities.

\subsubsection{On the Nature of Fitness}

First, there is the controversy over the nature of fitness, as a probabilistic magnitude. Biologists appeal to the notion of fitness in order to explain evolutionary changes:

\footnotetext{
18 A concept that is explicitly analogous, 'ecological drift', has been forged in community ecology by Hubbell (2001).
} 
differences in fitness between types (alleles or traits) in a given population are invoked to explain differences in the average offspring contribution of these types and accordingly changes in the proportions of these types in the population over time. Traditionally, the most common definition of fitness in biology is in terms of actual reproduction: fitness corresponds to the actual number of offspring left by an individual or type. There are a few problems with this actualist conception of fitness. First, it makes explanations invoking fitness differences circular. Under this definition, type $A$ being fitter than type $B$ means that type $A$ actually leaves a higher number of offspring than type $B$. Thus, the difference in fitness between type $A$ and type $B$, i.e. the difference between their actual frequencies, cannot explain the difference between the actual offspring contribution of type $A$ and of type $B$. Accounting for differences in frequency by differences in fitness provides a description rather than explanation of the observed changes in the population over time. The problem of circularity is an aspect of the famous 'tautology problem' raised by Darwin's critics: if evolution by natural selection is the 'survival of the fittest', and there is no independent way of assessing who are the fittest, and accordingly the fittest is defined in terms of actual survival and reproduction, then evolution by natural selection is a tautology. Second, the actualist conception of fitness implies that genetically and phenotypically identical twins may have different fitness just because of different circumstances that are irrelevant from the perspective of evolutionary biology, like accidental fire. Accordingly, it is impossible to make a distinction between selection and drift (intuitively, drift accounts for changes in trait frequency - and accordingly in reproduction and death of individuals - due to factors that are not related to fitness).

The so called 'propensity interpretation of fitness' (PIF), proposed by Mills and Beatty (1979), is supposed to overcome the above difficulties. According to PIF, an individual's fitness is defined as the propensity (or the disposition, the ability, the tendency or the capability) of an individual organism to survive and reproduce in a particular environment and a particular population (Mills and Beatty 1979). PIF suggests that an individual's fitness can be explicated in probabilistic terms along a single-case propensity theory of probability. Accordingly, the actual number of offspring (which is sometimes called the 'actual' or 'realized' fitness) need not be equal to the expected number of offspring (its fitness). The actual number of offspring of an individual might depend on chance events, such as accidental fires. Yet, such events do not play a role in the predictions of evolutionary biology, which is focused on the expected number of offspring.

There are dozens of theories about fitness and a variety of notions of fitness, e.g. 'engineering fitness' (Burian 1983), 'Fisherian fitness' (Michod 2000), 'inclusive fitness' (Hamilton 1964) and 'invasion fitness' (used in the approach called 'adaptive dynamics', Metz et al. 1992). Interestingly, until recently PIF had achieved near-consensus status among philosophers (Brandon 1990; Burian 1983; Sober 1984), plausibly because it seemed to offer a promising solution to the above difficulties. But in the last decade there have been several critiques of PIF (see, for examples, Ariew and Lewontin 2004; Sober 2001; Beatty and Finsen 1989), and there is a flourishing industry of notions of fitness. 
One question about PIF concerns the exact nature of the probabilities that explicate it. In their contribution, Isabelle Drouet and Francesca Merlin analyze the notion of probability that underlines PIF, focusing on Mills and Beatty's (1979) original version. Mills and Beatty define individual fitness ('fitness, ') as the singlecase propensity of an individual organism to survive and reproduce in a particular environment and a particular population. Drouet and Merlin first argue that Mills and Beatty's explication of fitness ${ }_{1}$ is marred with ambiguities and that it is not entirely clear how PIF relates to the propensity interpretation of probability in general, and single-case propensity in particular. Further, Drouet and Merlin argue that fitness ${ }_{1}$ understood along the lines of PIF does not really explain actual differences in reproductive success: even if we assume that a disposition explains its manifestations and we consider fitness as a dispositional property of individual

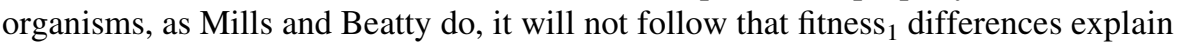
differences in actual reproductive success between individual organisms. Mills and Beatty (1979) and Beatty and Finsen (1989) define fitness of a type (fitness ${ }_{2}$ ) as the average fitness ${ }_{1}$ of the members of this type, where being a member of a type means having a certain allele or trait. That is, fitness ${ }_{2}$ is an average over the single-case propensities of individual organisms of that type to survive and reproduce. Drouet and Merlin point out that this definition also suffers from ambiguities and difficulties and they argue that fitness 2 explanations do not depend on how we interpret the probability in fitness ${ }_{1}$. In particular, Drouet and Merlin argue that fitness $_{2}$ explanations do not require that fitness ${ }_{1}$ be defined in terms of single-case

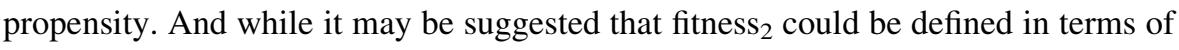
long-run propensities, Drouet and Merlin argue that such propensities would fail to serve the explanatory role they were designed to carry.

\subsubsection{Natural Selection and Chance}

A second main controversy concerning probabilities in biology concerns the nature of the chance that is involved in natural selection. Classically, natural selection is the main agent of evolution, and it acts on a Mendelian population that displays heritable variations due to mutations and recombinations (as Huxley summarized it in 1951 in a letter to Mayr $^{19}$ ). Intuitively, such mutations are random. But here the notion of randomness is vague. It may mean unpredictability in relation to environmental parameters, which is the common understanding of the term among evolutionary biologists. But it may also mean unpredictability simpliciter, and then the question is whether the mutations that are responsible for heritable variations are the result of indeterministic processes. Obviously, this question is central to the question whether evolutionary biology is a deterministic or indeterministic theory. Some authors hold that such mutations are indeterministic. Accordingly, they relate the nature of these mutations to QM and they strive to derive from the supposed indeterminism at the quantum level the random character of mutations (Brandon and

\footnotetext{
19 "[N]atural selection, acting on the heritable variation provided by the mutations and recombination of a Mendelian genetic constitution, is the main agency of biological evolution." The letter from Huxley to Mayr was intended to explain the general orientation of the book Evolution as a process, to which Mayr contributed (see Huxley et al. 1954).
} 
Carson 1996; Glymour 2001). Other authors object to this reasoning (Graves et al. 1999; Horan 1994; Rosenberg 2001). In any case, the interpretation of probabilities, especially probability distributions of mutations, plays a major role in this debate, which is naturally related to questions concerning the interpretation of probabilities in QM.

\subsubsection{Is Natural Selection a Cause?}

More recently, a third controversy concerning probability has divided philosophers of biology. Matthen and Ariew (2002) and Walsh et al. (2002) challenge the idea that selection and drift are 'forces' acting on population of alleles or traits-an idea that has floated around since the emergence of the field, and was theorized by Sober (1984). They argue that natural selection is not a cause but rather a statistical aggregate of the influences of ecological causes at the level of individual organisms. Thus, while natural selection could be used to predict evolution, it is not its cause. The upshot is that the causes of adaptation of organisms should be sought elsewhere at the individual level, e.g. in the individual developmental processes, and natural selection only describes or explains in some non-causal fashion the spread of adaptations. This so-called 'statisticalist' interpretation of selection has been developed and defended by Ariew, Matthen and Walsh (e.g. Walsh 2007, 2010; Matthen and Ariew 2009; Matthen 2009) and criticized by various authors (e.g. Millstein 2003; Brandon and Ramsey 2007; Bouchard and Rosenberg 2004; Abrams 2007; Huneman 2012). Here too, the debate is not wholly separated from physics. The statisticalists often advocate a 'thermodynamic' paradigm, where, in analogy to thermodynamic entropy, natural selection is a macroscopic, causally inert property. In the current issue, Denis Walsh provides a new clarification and defense of the statisticalist interpretation, whereas Marshall Abrams and Philippe Huneman analyze and criticize it.

An early line of critique of statisticalism is that it seems to deprive selection of any explanatory force (e.g. Bouchard and Rosenberg 2004). This critique is based on a causal account of explanation, which in turns is based on a firmly entrenched intuition that to explain a change one must cite its causes. Yet, Walsh argues that certain sciences are replete with non-causal, statistical explanations and that salient examples are found in theoretical population biology. Walsh aspires to explicate the nature of such explanations. In his contribution, he proposes the groundwork for a new account of statistical explanation and then applies it to theoretical population biology. Walsh suggests that an explanation serves two functions: metaphysical and cognitive. "The metaphysical function (roughly) is to identify a set of conditions in the world-the explanans-such that when they hold the explanandum does too. The cognitive function is that of enhancing our understanding of the occurrence of the explanandum". Inspired by recent accounts of mechanistic explanations, notably Woodward's (2003), Walsh proposes that the metaphysical function is satisfied by a change-relating invariance relation between the explanans and the explanandum. A relation between $X$ and $Y$ is change-relating just if a difference in the value of $X$ is associated with a difference in the value of $Y$, and the change-relating relation is invariant if it would continue to hold under changes in the values of $X$. 
Walsh proposes that the cognitive function of explanation-i.e. the enhancement of our understanding of the occurrence of the explanandum-is fulfilled by providing an elucidative description of the relation between the explanans and the explanandum. Since the explanation is supposed to be statistical and non-causal, Walsh requires that the elucidative description be one that cites only the statistical properties of the explanans (and "no activities or 'thick' causal concepts") and that the explanans do not cause the explanandum.

Like Fisher many decades ago, Walsh draws a parallel between finance and evolutionary biology. That is, Walsh draws a parallel between an example from investment portfolio theory and an effect in evolutionary biology which he calls the 'Gillespie effect': In small sub-populations a trait with a lower mean and lower variance will contribute on average a higher proportion of offspring to the next generation than a trait with a higher mean and higher variance, but in the population as a whole the fitness relations between the traits is reversed. Walsh argues that causalists fail to account for the Gillespie effect, and he proposes a statistical explanation of this effect. Walsh's statistical explanation proceeds in both the Gillepsie effect and the investment portfolio example in three steps, demonstrating that: (1) There is a change-relating invariance relation between the statistical properties — mean, variance and sample size — of explanans $X$ (e.g. a trait type) and an explanandum $Y$ (e.g. a relative frequency of offspring in the population). (2) There is an elucidating description that renders comprehensible the relation between the statistical properties of $X$ and $Y$. This elucidating description is strictly statistical: no causal concepts are employed. (3) $X$ do not cause $Y$.

In his contribution, Marshall Abrams argues that selection and drift should be characterized by causal probabilities. Causal probabilities do not constitute a specific interpretation of probability but rather classify a category of interpretations of probabilities. A causal probability distribution is an objective probability distribution that is realized by properties of a chance set-up. Alterations of such properties that change the probability distribution also change probabilistic patterns, i.e. what usually happens to frequencies, etc., in accordance with the change in the probability: those outcomes that are more probable occur more often, most of the time. For example, the causal probabilities of heads and tails are realized by the properties of a coin and a tossing device, and a change in the densities of the coin changes these probabilities and the corresponding probabilistic patterns. Based on this characterization, Abrams argues that the view that causal probabilities govern various systematic patterns in biological populations is the only one that fits common practices of simulating evolution in population genetics.

Recall that statisticalists argue that natural selection is an aggregate effect of individual interactions between organisms rather than an independent cause acting on populations. One of the consequences of this position is that it is difficult to make a distinction between selection and drift. Consider, for example, two traits, $A$ and $B$. Suppose that the fitness values of $A$ and $B$ are such that the expected frequency of each of these traits is 0.5 , but that actually the frequencies of $A$ and of $B$ turned out to be 0.6 and 0.4 , respectively. Assuming that selection accounts for the expected frequency and drift accounts for deviations from it, it seems meaningless to ask whether a particular realization of the trait $A$ is the result of selection or drift. Thus, 
the challenge is to establish a conceptual distinction between selection and drift (Matthen and Ariew 2002). This inscrutability of selection and drift appears to be ontological (rather than merely epistemic), as there seems to exist no information that could identify outcomes as pertaining to selection rather than drift. In his contribution, Philippe Huneman addresses this challenge. Based on a counterfactual account of causality, he argues that a conceptual difference between selection and drift could be established. The idea is basically the following. When there is selection, variations in the frequencies of traits are counterfactually dependent on the nature of the traits. When there is a drift, the increase in the frequency of traits is not counterfactually dependent on the nature of the traits. This idea could be formulated in terms of counterfactuals with a probabilistic consequent. For example, if the trait value 'blue eyes' had been different, the probability of the reproductive success of its carriers would have been different; where here the probability is to be interpreted as long-run propensity. This counterfactual entails that a change in the trait value 'blue eyes' would yield a change in the probabilities of the actual frequencies of the carriers and accordingly a change in the expected frequency of the class type 'blue-eyed individuals'. Thus, we have a conceptual distinction between selection and drift, which is based on an ontological difference between two kinds of processes.

Huneman makes a distinction between ontological inscrutability of selection and drift and epistemic opacity of selection and drift. The the epistemic opacity of selection and drift is related to our incomplete knowledge of selective pressures and is common in various cases, especially in small populations. When considering actual or hypothetical sets of replicates of various sizes of populations, drift and selection yield distinct statistical signatures, described in the paper. Huneman thus argues that research programs that seek to distinguish between the processes and effects of selection and the processes and effects of drift face only epistemic obstacles.

\subsection{On Probabilities and Probabilistic Reasoning in Classical Mechanics and Bohmian Mechanics}

Questions concerning the interpretation of probability have been a major focus in the philosophy of physics. This is particularly true of QM and CSM. Three of the contributions to this volume focus on foundational questions concerning probabilities in these theories. In what follows in this section, we briefly review the background for these questions and the main highlights of the contributions.

\subsubsection{On the Interpretation of Probabilities in Quantum Mechanics}

There are various questions that are relevant for the interpretation of probability in QM. One question is concerned with what QM wavefunctions represent. Do they represent the states of physical systems or rather knowledge or ignorance about these states? The interpretation of QM wavefunctions as states of systems is traditionally associated with objective probability, though there is a controversy concerning whether QM probabilities should be interpreted along frequency 
theories, ${ }^{20}$ propensity theories (Popper 1957; Redhead 1987; Krips 1989) or THOC (Frigg and Hoefer 2007). Different interpretations of probability may be suitable for different interpretations of QM.

The interpretation of QM wavefunctions as states of knowledge or ignorance has been recently aligned with subjectiveltheories of probability (see Sect. 3). Another way to interpret QM wavefunctions as reflecting ignorance is to take them to represent ensembles of systems with different states. It is noteworthy, however, that due to quantum entanglement the interpretation of QM wavefunctions along these lines is a rather delicate matter, as not all QM wavefunctions can be considered as proper mixtures of ensemble of systems with different states (d'Espagnat 1976).

Another question that plays an important role in the interpretation of probabilities in $\mathrm{QM}$ is whether the quantum realm is indeterministic or deterministic. For example, single-case propensity is a natural candidate for the interpretation of probabilities in indeterministic interpretations of QM, but not in deterministic interpretations, such as Bohmian mechanics. In their contribution, Meir Hemmo and Orly Shenker discuss the interpretation of probability in Bohmian mechanics (BM). In BM, the QM wavefunction of a system and its position configuration jointly determine its future trajectory and the outcome of any measurement on it (as long as the outcome is recorded in the position of some physical system, as in any practical measurement). BM reproduces the statistical predictions of standard QM (the Born rule) by postulating that the probability distribution over all possible position configurations of a system at a given time is given by the absolute square of its QM wavefunction at that time. The natural interpretation of this distribution is along subjective theories of probability, understanding this postulate as providing a measure of ignorance over all the possible position configurations. But, Dürr et al. (1992), notable supporters of BM, have opted to avoid such interpretation and explained the empirical adequacy of BM by an appeal to the notion of a typical initial position configuration. Durr, Goldstein and Zanghi (DGZ) argue that for every initial universal wavefunction and for any typical initial global configuration, the probability distribution over the position of subsystems of the universe is given by the absolute square of the effective wavefunctions of these systems (when the effective wavefunctions exist). ${ }^{21}$ Here typicality is defined relative to the initial universal wavefunction-typical initial global configurations constitute the overwhelming majority of initial configurations relative to the measure induced by the absolute square of the initial universal wavefunction-and the probability distributions prescribed by effective wavefunctions in typical initial global configurations may be interpreted along long-run frequency or long-run propensity theories of probability.

In their contribution, Hemmo and Shenker argue that since the notion of typicality is supposed to be non-probabilistic, it is unclear why a condition that obtains for most initial conditions (relative to the absolute square of the universal

\footnotetext{
${ }^{20}$ As Landsman (2009, p. 60) notes, the pragmatic attitude taken by most physicists is that the probabilities in Born's rule are to be interpreted as long-run frequencies.

21 Roughly, an effective wavefunction of a system exists when 'enough' decoherence has occurred between the system and its environment (see Dürr et al. 1992; Callender 2007).
} 
wavefunction) should be taken to obtain for a given system. That is, the problem is to justify this measure of typicality in a non-circular way, and Hemmo and Shenker argue that DGZ fail to provide such a justification. In his contribution, Berkovitz (2015, Sect. 3.2.4) argues that this critique also reflects on the credentials of finite long-run propensity interpretations of the distributions prescribed by effective wavefunctions.

\subsubsection{On the Interpretation of Probabilities and Probabilistic Reasoning in Classical Statistical Mechanics}

Hemmo and Shenker also criticize the application of the typicality approach in CSM. Recall (Sect. 3) that a major problem in the foundations of CSM is to explain why isolated macroscopic physical systems never seem to evolve to states of lower entropy and that the standard reply-that thermodynamic behavior is highly probable-raises questions concerning the interpretation of probability and the justification of the Lebesgue measure as the choice of the measure for calculating probabilities of macrostates. Rather than appealing to probabilistic considerations, the typicality approach has opted to justify the Lebesgue measure by arguing that it is natural. The term 'natural' is rather vague and Hemmo and Shenker consider various specifications of it and argue that they either fail to justify the Lebesgue measure or implicitly rely on some probabilistic assumptions that need to be justified. Thus, they conclude that typicality considerations are not justified as grounding probability statements in CSM. Hemmo and Shenker propose that the choice of the probability measure in CSM should be made on the basis of the dynamics of microstates and empirical considerations in the form of relative frequencies of the macrostates found in experience. In this alternative approach, the probability distribution over the initial conditions is derivable from the transition probabilities of macrostates, which are based on observed frequencies of macrostates. These transition probabilities are supposed to reflect 'objective ignorance', namely ignorance that is based on objective facts: the dynamics that govern the trajectories in the phase space and the partition of the phase space into macrostates. Hemmo and Shenker argue that the resulting measure need not be the Lebesgue measure, though this measure may be the most convenient among the measures that are compatible with the relative frequencies of macrostates. The upshot is that the probability measure in CSM is justified on empirical grounds and convenience.

Recall (Sect. 3) that since CSM is a deterministic theory it is natural to interpret probabilities of microstates and macrostates of systems as reflecting our ignorance about them but that this interpretation raises the question of how our ignorance could explain the objective thermodynamic behavior of systems. This question motivates the search for an objective interpretation of the probabilities in CSM. In some theories of objective probability, non-trivial probabilities exist only in indeterministic worlds, and accordingly they cannot serve as an interpretation of the probabilities in CSM. Single-case propensity theories and Lewis' (1986, chap. 19) THOC are notable examples. In their contribution, Roman Frigg and Carl Hoefer propose a new version of THOC and argue that it is suitable for the interpretation of the probabilities in CSM. Like in Lewis' theory (see Sect. 2), in this new theory: 
chances constrain subjective probabilities according to the principal principle (PP); chances have the values that are assigned by rules which are part of a best system (BS) of laws and rules, striking as good a balance as the actual events will allow of simplicity, strength and fit; and (given the criteria of simplicity, strength and fit) chances supervene on everything that actually happens, the so-called 'Humean mosaic'. But unlike in Lewis' theory, in Frigg and Hoefer's theory non-trivial chances may exist in deterministic universes. Following Hoefer (2007, 558-559), Frigg and Hoefer argue that Lewis' commitment to the existence of non-trivial chances only in indeterministic universes results from his view that all chances follow from the laws of fundamental physics. (This is reflected in Lewis' reading of PP, where fundamental laws are always admissible information and accordingly chances in deterministic universes are trivial.) Frigg and Hoefer reject this view. They maintain that rules for assigning chances can be formulated in terms pertaining to different levels of discourse, such as microphysics, macrophysics, chemistry, genetics, mechanical engineering and meteorology, and that rules from all these disciplines have equal right to be considered for inclusion in a BS package. They then apply this revised version of THOC to CSM. There are two major traditions in CSM, sometimes seen as two different theories: one originates in the work of Gibbs and the other in the work of Boltzmann (Frigg 2008). Frigg and Hoefer argue that the probabilities in Boltzmannian CSM can be interpreted along their version of THOC.

The problem of reconciling the expressly time-asymmetric behavior of irreversible thermodynamic processes with the underlying time-symmetric dynamics of CM systems can be related to the question of whether we can take our memory and records to be reliable. Suppose, for example, a glass of water with a half-melted ice cube in it, suitably isolated from its environment. Given this present macrostate, we can follow the system's underlying CM dynamics to predict and retrodict its future and past macrostate, respectively. Based on the uniform probability distribution over the system's present macrostate and the CM dynamics that purportedly underlie thermodynamic systems, it would appear that (contrary to our best recollections) such a non-equilibrium state popped into existence as an enormously improbable fluctuation from a past equilibrium macrostate. If we take our memories and records of past events to be describable in CSM terms, the above concerns seem to apply equally well to them. Just as we can retrodict that the ice cube arose as a spontaneous fluctuation from an equilibrium state, so can we retrodict that our present memories of the ice cube most likely arose out of a spontaneous fluctuation. More generally, taking records as CSM systems, it would appear that all our records arose spontaneously from equilibrium states and should not be taken as veridical (Albert 2000, p. 115).

Albert suggests that the past hypothesis - the claim that the universe found itself in a low entropy state early in its history-could meet the above reversibility objection, and that this hypothesis can be supported on inductive grounds (ibid., p. 94). But, as Daniel Parker points out in his contribution, this justification of the past hypothesis presupposes that we can trust our memories, which is precisely what is at issue. Rather than attempting to justify the past hypothesis or some other close cousins of it, Parker challenges an implicit presupposition of the reversibility objection concerning records: namely, that records (and memories) of events, 
insofar as they are recognized as such, are records solely in virtue of their CSM properties. He argues that the representational content of a record is conceptually independent of its thermodynamic state. The content of a record does not evolve according to any natural dynamics, and is not susceptible to the reversibility objection. A correlation between a record and the recorded system is not described by a physical correlation between the two systems, but rather by a correlation between the recorded system and the representational content of the record. Based on this conception of records, Parker argues that in many cases, our retrodictionsbased solely on the present macrostate of the universe (including the representational content of our records), the standard probability distribution over the microstates that are compatible with that macrostate and the laws of motion-speak to the veracity of our records and the common causal structure of record generating processes.

\subsection{On Probabilistic Independence in Models of 'Reducible' Probabilities}

Probability also plays a central role in causal inference in biology and physics. For example, Bell's (1987) celebrated no-go theorem for local hidden-variables quantum theories is based on the assumption that in such theories, probabilistic dependence between measurement outcomes which are causally independent of each other is due to a common cause, and that the common cause (the 'hidden variable') renders the outcomes probabilistically independent. More generally, it is common to assume that a probabilistic dependence between events must have a causal explanation. Accordingly, inferences, hypotheses and suppositions about probabilistic independence between events are often based on the assumption that the events are causally unrelated to each other, in the sense of having neither a direct causal connection nor a common cause. In his contribution, Michael Strevens discusses circumstances under which events are probabilistically independent even though they are causally related. Strevens points out that while the existence of a common cause frequently establishes a probabilistic dependence between events, events with a common cause are often treated as probabilistically independent. For example, in CSM the position and velocity of a gas molecule are treated as probabilistically independent of the positions and velocities of the other molecules, even though they are determined entirely by the past interactions with those other molecules. Similarly, in population genetics an instance of gene replication is treated as probabilistically independent of the other instances, even though replication is determined in all cases by events in a single, densely causally connected ecosystem.

Strevens thinks that when probabilities are fundamental and accordingly 'irreducible', as QM probabilities are often conceived, the question whether probabilistic independence could be justified in spite of causal dependencies and causal commonalities may be settled by an appeal to fundamental laws of nature. However, in the great majority of scientific and everyday cases-such as gambling setups, actuarial models, CSM and evolutionary biology-probabilities are not irreducible. An interesting philosophical project is to state the conditions under which causally related events may be assumed to be probabilistically independent relative to the reducible physical probability distributions attributed to them by 
scientific models and theories. The extent of this project is vast, and in his contribution Strevens addresses a small piece of it. He derives the conditions for independence among the outcomes of coin tosses that are causally connected in two ways: (1) two consecutive coin tosses made by a single croupier and (2) two tossed coins with a midair collision.

\subsection{On the Conceptual Foundations of Propensity Theories of Probability}

Propensity theories of probability are among the main objective interpretations of probability, and they have been applied in both biology and physics. Yet, the conceptual foundations of these theories are far from clear or unproblematic, and clarifying them is an important aspect of the interpretation and application of probability in science. Further, propensity theories have been subjected to various objections. For example, it has been argued that it is difficult to explain why propensities should satisfy the probability calculus (Eagle 2004; Hájek 2012) and even worse that propensities are at odd with this calculus (Humphreys 1985, 2004), that various explications of propensities are circular and accordingly not informative (Mellor 1971; Rosenthal 2006) and that single-case propensities are non-scientific (Gillies 2000b).

The tendency in the literature is to overlook the variety of propensity theories and the substantial differences between them. In the first part of his contribution, Joseph Berkovitz provides a detailed, critical review of the main propensity theories, and in the second part he addresses the objections above. Berkovitz argues that the claim that propensity theories are at odds with the probability calculus-the so-called 'Humphreys' paradox' - is based on the inadequate representation of propensities as standard conditional probabilities. He proposes that the fundamental object of propensity theories is a non-standard conditional probability, which may be thought of as a conditional with a probabilistic consequent (albeit not any of the standard ones), and on the basis of this proposal he shows that propensities do satisfy the probability calculus. Berkovitz also suggests two ways to motivate the probability calculus in the context of propensity theories: the first appeals to the idea that there is a mathematical constitution of physical facts and that the axioms of probability are part of the ontological nature of propensities, and the second appeals to the relations between propensities and subjective probabilities. Next, Berkovitz analyzes the relations between propensities and frequencies and propensities and subjective probabilities and demonstrates how these relations could shed light on the nature of propensities and their time-evolution. Finally, he argues that the claim that single-case propensities are non-scientific is based on misconceptions about the way in which hypotheses about propensities are formed and evaluated.

Berkovitz's conclusion is that while propensity theories face various challenges, none of these challenges undermines their viability as prospective interpretations of probability in science in general, and in biology and physics in particular.

Acknowledgments For comments on earlier versions of this paper, we are grateful to Noah Stemeroff and Marshal Abrams. 


\section{References}

Abrams, M. (2007). How do natural selection and random drift interact? Philosophy of Science, 74(5), $666-679$.

Abrams, M. (2012). Mechanistic probability. Synthese, 187(2), 343-375.

Abrams, M. (2015). Probability and manipulation: Evolution and simulation in applied population genetics. Erkenntnis (this volume).

Albert, D. (2000). Time and chance. Cambridge, MA: Harvard University Press.

Ao, P. (2005). Laws in Darwinian evolutionary theory. Physics of Life Reviews, 2(2), 117-156.

Ariew, A., \& Lewontin, R. C. (2004). The confusions of fitness. British Journal for the Philosophy of Science, 55(2), 347-363.

Barton, N., \& Coe, J. B. (2009). On the application of statistical physics to evolutionary biology. Journal of Theoretical Biology, 259(2), 317-324.

Beatty, J., \& Finsen, S. (1989). Rethinking the propensity interpretation: A peek inside the Pandora's box. In M. Ruse (Ed.), What the philosophy of biology is today: Essays for David Hull (pp. 17-30). Dordrecht: Kluwer.

Bell, J. S. (1987). Speakable and unspeakable in quantum mechanics. Cambridge: CUP.

Berkovitz, J. (2012). The world according to de Finetti: On de Finetti's theory of probability and its application to quantum mechanics. In Y. Ben Menachem \& M. Hemmo (Eds.), Probability in physics, The Frontiers Collection (pp. 249-280). New York: Springer.

Berkovitz, J. (2014). On de Finetti's instrumentalist philosophy of probability. A paper presented at the Munich Center for Mathematical Philosophy. https://cast.itunes.uni-muenchen.de/vod/clips/ uXGOLuIMeX/quicktime.mp4.

Berkovitz, J. (2015). The propensity interpretation: A re-evaluation. Erkenntis (this volume).

Bolzano, B. (1973). Theory of science, edited, with an introduction by J. Berg, translated from German by Burnham Terrell. Dordrecht and Boston: D. Reidel.

Bouchard, F., \& Rosenberg, A. (2004). Fitness, probability and the principles of natural selection. British Journal for Philosophy of Science, 55(4), 693-712.

Brandon, R. (1990). Adaptation and environment. Cambridge MA: MIT Press.

Brandon, R., \& Carson, S. (1996). The indeterministic character of evolutionary theory: No "no hidden variables' proof but no room for determinism either. Philosophy of Science, 63(3), 315-337.

Brandon, R., \& Ramsey, G. (2007). What's wrong with the emergentist statistical interpretation of natural selection and random drift? In M. Ruse \& D. Hull (Eds.), The Cambridge companion to philosophy of biology (pp. 66-84). Cambridge: CUP.

Bub, J. (1975). Popper's propensity interpretation of probability and quantum mechanics. In G. Maxwell \& R. M. Anderson (Eds.), Induction, probability and confirmation. Minnesota studies in the philosophy of science (Vol. 6, pp. 416-429). Minneapolis: University of Minnesota Press.

Bub, J., \& Pitowsky, I. (1985). Critical notice of Karl Popper's postscript to the logic of scientific discovery. Canadian Journal of Philosophy, 15(3), 539-552.

Burian, R. (1983). Adaptation. In M. Grene (Ed.), Dimensions of Darwinism (pp. 286-314). Cambridge: CUP.

Callender, C. (2007). The emergence and interpretation of probability in Bohmian mechanics. Studies in History and Philosophy of Modern Physics, 38(2), 351-370.

Carnap, R. (1950). Logical foundations of probability. Chicago: The University of Chicago Press, 2nd edition with modifications 1962.

Carnap, R. (1952). The continuum of inductive methods. Chicago: University of Chicago Press.

Caves, C. M., Fuchs, C. A. \& Schack, R. (2002). Quantum probabilities as Bayesian probabilities. Physical Review 65(2), 1-6. quant-ph/0106133.

Caves, C. M., Fuchs, C. A., \& Schack, R. (2002b). Unknown quantum states: The quantum de Finetti representation. Journal of Mathematical Physics, 43(9), 4537-4559.

Caves, C. M., Fuchs, C. A., \& Schack, R. (2007). Subjective probability and quantum certainty. Studies in History and Philosophy of Modern Physics 38(2), 255-274. quant-ph/0608190.

d'Espagnat, B. (1976). Conceptual foundations of quantum mechanics (2nd ed.). Boston: AddisonWesley.

de Finetti, B. (1972). Probability, induction and statistics. New York: Wiley.

de Finetti, B. (1974a). Theory of probability (Vol. 1). New York: Wiley.

de Finetti, B. (1974b). Theory of probability (Vol. 2). New York: Wiley. 
Dickey, J. M., Eaton, M. L., \& Sudderth, W. D. (2009). De Finetti coherence and logical consistency. Notre Dame Journal of Formal Logic, 50(2), 133-139.

Drouet, I., \& Merlin, F. (2014). The propensity interpretation of fitness and the propensity interpretation of probability. Erkenntnis (this volume).

Dürr, D., Goldstein, S., \& Zanghi, N. (1992). Quantum equilibrium and the origin of absolute uncertainty. Journal of Statistical Physics, 67(5/6), 843-907.

Eagle, A. (2004). Twenty-one arguments against propensity analyses of probability. Erkenntnis, 60(3), 371-416.

Ellis, R. L. (1849). Remarks on the fundamental principle of the theory of probability. Transactions of the Cambridge Philosophical Society, VIII, 1-6.

Fetzer, J. H. (1974). A single case propensity theory of explanation. Synthese, 28(2), 171-198.

Fetzer, J. H. (1981). Scientific knowledge: Causality, explanation and corroboration. Dordrecht: Reidel.

Fetzer, J. H. (1982). Probabilistic explanations. In P. Asquith \& T. N. Nickles (Eds.), PSA 1982 (Vol. 2, pp. 194-207). East Lansing, MI: Philosophy of Science Association.

Fisher, R. A. (1930). The genetical theory of natural selection. Oxford: OUP.

Frigg, R. (2008). A field guide to recent work on the foundations of statistical mechanics. In D. Rickles (Ed.), The Ashgate companion to contemporary philosophy of physics (pp. 99-196). London: Ashgate.

Frigg, R., \& Hoefer, C. (2007). Probability in GRW theory. Studies in History and Philosophy of Modern Physics, 38(2), 371-389.

Frigg, R., \& Hoefer, C. (2013). The best Humean system for statistical mechanics. Erkenntnis (this volume).

Galavotti, M. C. (2005). Philosophical introduction to probability. Stanford, California: CSLI Publications.

Giere, R. (1973a). Review of Mellor's (1971) The Matter of Chance. Ratio, 15, 149-155.

Giere, R. (1973b). Objective single-case probabilities and the foundations of statistics. In P. Suppes, et al. (Eds.), Logic, methodology and philosophy of science IV (pp. 467-483). Amsterdam, London: North-Holland Publishing Company.

Giere, R. (1975). The epistemological roots of scientific knowledge. In G. Maxwell \& R. M. Anderson Jr (Eds.) Induction, probability, and confirmation, Minnesota studies in the philosophy of science. (Vol. VI, pp. 212-261). Minneapolis: University of Minnesota Press.

Giere, R. (1979). Propensity and necessity. Synthese, 40(3), 439-451.

Gillies, D. (2000a). Philosophical theories of probability. London: Routledge.

Gillies, D. (2000b). Varieties of propensities. British Journal for the Philosophy of Science, 51(4), $807-835$.

Glymour, B. (2001). Selection, indeterminism, and evolutionary theory. Philosophy of Science, 68(4), 518-535.

Graves, L., Horan, B. L., \& Rosenberg, A. (1999). Is indeterminism the source of the statistical character of evolutionary theory? Philosophy of Science, 66(1), 140-157.

Hacking, I. (1965). Logic of statistical inference. Cambridge: CUP.

Hájek, A. (2003). What conditional probability could not be? Erkenntnis, 137(3), 273-323.

Hájek, A. (2012). Interpretations of probability. In Edward N. Zalta (Ed.) The Stanford encyclopedia of philosophy. Summer 2012 Edition forthcoming. http://plato.stanford.edu/archives/sum2012/entries/ probability-interpret/.

Hamilton, W. D. (1964). The genetic evolution of social behavior. Journal of Theoretical Biology, 7(1), $1-16$.

Hemmo, M., \& Shenker, O. (2014). Probability and typicality in deterministic physics. Erkenntnis (this volume).

Hoefer, C. (2007). The third way on objective probability: A sceptic's guide to objective chance. Mind, 116(463), 549-596.

Horan, B. (1994). The statistical character of evolutionary biology. Philosophy of Science, 61(1), 76-95.

Howson, C. (2008), De Finetti, countable additivity, consistency and coherence. British Journal for the Philosophy of Sciece, 59(1), 1-23.

Hubbell, S. (2001). The unified neutral theory of biodiversity and biogeography. Princeton: Princeton University Press.

Humphreys, P. (1985). Why propensities cannot be probabilities. Philosophical Review, 94(4), 557-570.

Humphreys, P. (2004). Some considerations on conditional chances. British Journal for the Philosophy of Science, 55(4), 667-680. 
Huneman, P. (2012). Natural selection: A case for the counterfactual approach. Erkenntnis, 76(2), 171-194.

Huneman, P. (2014). Inscrutability and the opacity of natural selection and random genetic drift: Distinguishing epistemic and metaphysical aspects. Erkenntnis (this volume).

Huxley, J., Hardy, H. C., \& Ford, E. B. (Eds.). (1954). Evolution as a process. London: George allen. Jaynes, E. T. (1957). Information theory and statistical mechanics. Physical Review, 106(4), 620-630. Jeffreys, H. (1961). Theory of probability (3rd ed.). Oxford: Clarendon Press.

Johnson, W. E. (1932). Probability: The relations of proposal to supposal; Probability: Axioms; Probability: The deductive and the inductive problems. Mind, 41, 1-16, 281-296, 409-423.

Keynes, J. M. (1921). Treatise on probability. London: Macmillan.

Kolmogorov, A. N. (1933/1950). Foundations of the theory of probability (N. Morrison, Trans.). New York: Chelsea Publishing.

Krips, H. (1989). A propensity interpretation of quantum probabilities. The Philosophical Quarterly, 39(156), 308-333.

Landsman, N. P. (2009). Born rule and its interpretation. In D. Greenberger, K. Hentschel, \& F. Weinert (Eds.), Compendium of quantum mechanics. Berlin: Springer.

Lewis, D. (1986). Philosophical papers (Vol. 2). Oxford: OUP.

Maher, P. (2010). Explication of inductive probability. Journal of Philosophical Logic, 39(6), 593-616.

Matthen, M. (2009). Drift and 'statistically abstractive explanation'. Philosophy of Science, 76(4), $464-487$.

Matthen, M., \& Ariew, A. (2002). Two ways of thinking about natural selection. Journal of Philosophy, $49(2), 55-83$.

Matthen, M., \& Ariew, A. (2009). Selection and causation. Philosophy of Science, 76(2), 201-224.

Mellor, D. H. (1969). Chance-I. In D. H. Mellor \& J. Walting (Eds.), Proceedings of the Aristotelian Society, supplementary volumes (Vol. 43, pp. 11-36).

Mellor, D. H. (1971). The matter of chance. Cambridge: Cambridge University Press.

Mellor, D. H. (1995). The facts of causation. London: Routledge.

Metz, J. A. J., Nisbet, R. M., \& Geritz, S. A. H. (1992). How should we define fitness for general ecological scenarios. Trends in Ecology \& Evolution, 7(6), 198-202.

Michod, R. E. (2000). Darwinian dynamics (New ed.). Princeton: Princeton University Press.

Miller, D. (1991). Single-case probabilities. Foundations of Physics, 21(12), 1501-1516.

Miller, D. (1994). Critical rationalism: A restatement and defence. La Salle, Ill: Open Court.

Mills, S., \& Beatty, J. (1979). The propensity interpretation of fitness. Philosophy of Science, 46(2), 263-286.

Millstein, R. (2003). Interpretations of probability in evolutionary theory. Philosophy of Science, 70(5), 1317-1328.

Mises, von R. (1928). Probability, statistics and truth. (2nd rev. English edn.). London: George Allen \& Unwin (1961).

Morrison, M. (2002). Modelling populations: Pearson and Fisher on Mendelism and biometry. British Journal for the Philosophy of Science, 53(1), 39-68.

Peirce, C. S. (1910/2011). A note on the doctrine of chances. In J. Buchler (Ed.), Philosophical writings of pierce (pp. 164-172). New Dover, 2011.

Pitowsky, I. (2003). Betting on the outcomes of measurements: A Bayesian theory of quantum probability. Studies in History and Philosophy of Modern Physics, 34(3), 395-414.

Popper, K. R. (1957). The propensity interpretation of the calculus of probability, and quantum theory. In S. Körner (Ed.), Observation and interpretation in the philosophy of physics (pp. 65-70). New York: Dover.

Popper, K. R. (1959). The propensity interpretation of probability. British Journal for the Philosophy of Science, 10(37), 25-42.

Popper, K. R. (1967). Quantum mechanics without 'the observer'. In M. Bunge (Ed.), Quantum theory and reality (pp. 7-44). New York: Springer.

Popper, K. R. (1990). A world of propensities. Bristol: Thoemmes.

Psillos, S. (1999). Scientific realism. London: Routledge.

Ramsey, F. P. (1926/1990). Truth and probability. Reprinted In D. H. Mellor (Ed.), Frank Ramsey: Philosophical papers. Cambridge: CUP, 1990.

Redhead, M. (1987). Incompleteness, nonlocality and realism: A prolegomenon to the philosophy of quantum mechanics. Oxford: OUP.

Reichenbach, H. (1949). The theory of probability. Berkeley: University of California Press. 
Rosenberg, A. (1994). Instrumental biology or the disunity of science. Chicago: University of Chicago Press.

Rosenberg, A. (2001). Discussion note: Indeterminism probability, and randomness in evolutionary theory. Philosophy of Science, 68(4), 536-544.

Rosenthal, J. (2006). Karl Popper's propensity interpretation of probability. In I. Jarvie, K. Milford, \& D. Miller (Eds.), Karl Popper: A centenary assessment (Vol. 3). Aldershot, UK: Science.

Rosenthal, J. (2010). The natural-range conception of probability. In G. Ernst \& A. Hüttemann (Eds.), Time, chance and reduction: Philosophical aspects of statistical mechanics (pp. 71-91). Cambridge: CUP.

Rosenthal, J. (2012). Probabilities as ratios of ranges in initial-state spaces. Journal of Logic, Language and Information, 21(2), 217-236.

Sella, G., \& Hirsh, A. E. (2005). The application of statistical physics to evolutionary biology. Proceedings of the National Academy of Sciences USA, 102, 9541-9546.

Sober, E. (1984). The nature of selection. Cambridge, MA: MIT Press.

Sober, E. (2001). The two faces of fitness. In R. S. Singh, C. B. Krimbas, D. P. Paus, \& J. Beatty (Eds.), Thinking about evolution: Historical, philosophical, and political perspectives (pp. 309-321). Cambridge: CUP.

Strevens, M. (2011). Probability out of determinism. In C. Beisbart \& S. Hartmann (Eds.), Probabilities in physics (pp. 339-364). Oxford: OUP.

Strevens, M. (2014). Stochastic independence and causal connection. Erkentnnis (this volume).

Suarez, M. (2013). Propensities and pragmatism. The Journal of Philosophy, 110(2), 61-92.

Venn, J. (1866). The logic of chance. London: Macmillan.

Vineberg, S. (2011). Dutch Book Arguments. In Edward N. Zalta (Ed.), The Stanford encyclopedia of philosophy. Summer 2011 Edition. http://plato.stanford.edu/archives/sum2011/entries/dutch-book/.

von Kries, J. (1886). Die Principien der Wahrscheinlichkeitsrechnung. Tübingen: Mohr Siebeck.

Walsh, D. M. (2007). The pomp of superfluous causes: The interpretation of evolutionary theory. Philosophy of Science, 74(3), 281-303.

Walsh, D. M. (2010). Not a sure thing: Fitness probability and causation. Philosophy of Science, 77(2), $141-171$.

Walsh, D. M. (2014). Variance, invariance and statistical explanation. Erkenntnis (this volume).

Walsh, D. M., Lewens, T., \& Ariew, A. (2002). Trials of life: Natural selection and random drift. Philosophy of Science, 69(3), 452-473.

Woodward, J. (2003). Making things happen: A theory of causal explanation. Oxford: OUP. 